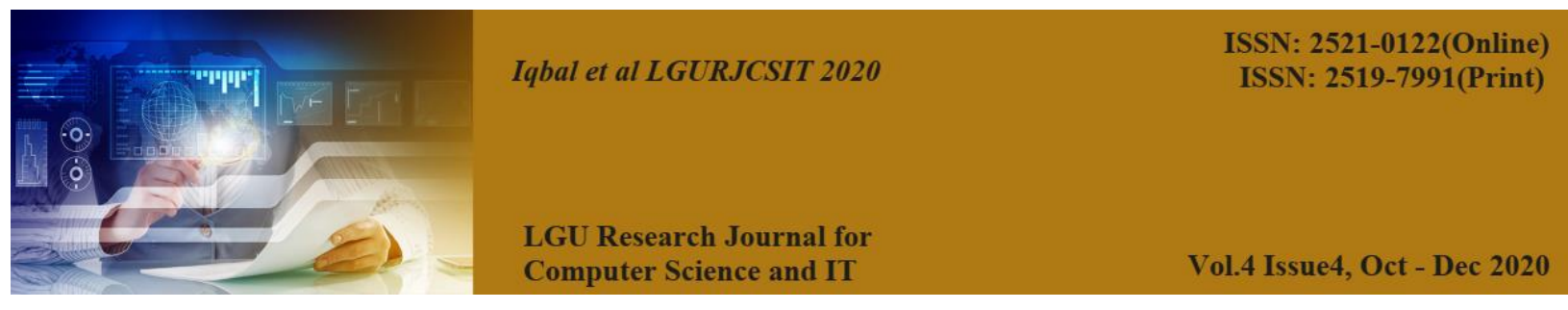

\title{
A Concurrence Study on Interoperability Issues in IoT and Decision Making Based Model on Data and Services being used during Inter-Operability
}

Muhammad Raza Naqvi ${ }^{1}$, Muhammad Waseem Iqbal ${ }^{1}$, Syed Khuram Shahzad ${ }^{2}$,

Iqra Tariq ${ }^{3}$, Marium Malik ${ }^{4}$,Faseeha Ehsan ${ }^{5}$, Natash Ali Mian ${ }^{6}$, Nadia Tabassum ${ }^{7}$

1,6 Department of Computer Science, The Superior College, Lahore, Pakistan.

${ }^{1,4}$ Department of Software Engineering, The Superior College, Lahore, Pakistan.

${ }^{2}$ Department of Informatics \& Systems, University of Management and Technology, Lahore, Pakistan.

${ }^{3}$ Department of Computer Science, The University of Lahore Gujrat Campus, Gujrat, Pakistan.

${ }^{6}$ School of Computer and Information Technology, Beaconhouse National University,Lahore, Pakistan

${ }^{7}$ Department of Computer Science,Virtual University, Pakistan

${ }^{1}$ waseem.iqbal@ superior.edu.pk

\section{Abstract:}

The Internet-of-Things (IoT) has become an important topic among researchers owing to its potential to change the way we live and use smart devices. In recent years, many research work found in the world are interrelated and convey via the existing web structure which makes a worldwide system called IoT. This study focused on the significant improvement of answers for a wider scope of gadgets and the Internet of Things IoT stages in recent years. In any case, each arrangement gives its very own IoT framework, gadgets, APIs, and information configurations promoting interoperability issues. These issues are the outcome of numerous basic issues, difficulty to create IoT application uncovering cross-stage, and additionally cross-space, trouble in connecting non-interoperable IoT gadgets to various IoT stages, what's more, eventually averts the development of IoT innovation at an enormous scale. To authorize consistent data sharing between various IoT vendors, endeavors by a few academia, industrial, and institutional groups have accelerated to support IoT interoperability. This paper plays out a far-reaching study on the cutting-edge answers for encouraging interoperability between various IoT stages. Likewise, the key difficulties in this theme are introduced.

Keywords: Internet of things IoT, IoT Interoperability, Semantic Interoperability.

\section{INTRODUCTION}

Interoperability is the capacity of several interconnected IT systems and applications 
to interchange data precisely, efficiently, and reliably. Internet of Things (IoT), first began by Kevin Ashton around 1999 has as of late been a rising innovation in a wide scope of areas [1]. The starring role of the Internet has transformed because of the evolution of billions of physical devices connected via the Internet around the globe [2]. This idea characterizes a mechanical upset were mechanical as well as virtual things are associated with further things and to the present Internet framework. IoT is characterized as a basic worldwide system with designed characteristics dependent its typical as interoperable correspondence pacts where physical what's more, virtual things have characters, physical traits, and virtual characters and develop acute interfaces, and are faultlessly synchronized into the network [3]. IoT is dynamic by its very nature. For companies to survive, their business models will have to be dynamic as well. In the world of IoT, fast and flexible is the norm, and a company's culture, processes, tools, and technologies must be built on a foundation of rapid, continuous innovation. A wealth of keen associated gadgets and stages have been incorporated in a wide scope of utilizations like trade, social insurance, horticulture, utilities, vitality, transportation, mechanical control, and structures. From the perspective of the IoT suppliers', the absence of interoperability implies that specialist organizations are bound to the use IoT gadget or programming obtainable by a solitary supplier and must follow that and bound to use the instructions provided by the IoT supplier, which may arise the probable danger of greater activity budget later on, just as item usefulness and soundness issues. The incongruence between various IoT stages ensures the earth of IoT stage suppliers briefly until the IoT market grows increasingly develop. Specifically, it is in all respects expensive for little organizations to help.
Internet of Things (IoT) devices are prone to heterogeneity due to the communication technology, protocol variations, formats of data, and varied semantics. The models of semantics facilitate the meaningful exchange of information between heterogeneous devices making it a significant approach to provide service over distinct IoT platforms. Heterogeneous interfaces of every assorted stage. From the point of view of use designers, the contradiction between IoT stages brings about adjusting their application to the stage explicit Application Programming Interface and data models of each unique stage, which averts cross-stage, for example, applications which work on different stages and cross-domain application improvement, for example, applications which consolidate various areas. The significance of the interoperability issues in IoT has been stressed by academia and industrial researchers. The business endeavors to take up interoperability issues of IoT over institutionalization. A few endeavors have developed to build up norms for giving interoperability between IoT gadgets, systems, administrations, information arrangements possessed by various suppliers. The European Union has additionally as of late financed a few researches extends under the $\mathrm{H} 2020$ program concentrating upon the alliance of IoT stages. In any case, it might take quite a while before the related measures are completely settled upon and acknowledged, if at any point. To determine this challenge, analysts in individually scholarly community and industry have been building up a rundown of creative answers for interoperability and heterogeneity in various IoT frameworks. This paper gives a thorough report on IoT interoperability, what's more, presents interoperability characterization. Scientific classification of interoperability in IoT is formulated as alternate points of view to gadget interoperability, arrange 
interoperability, linguistic interoperability, semantic interoperability, and stage interoperability. Besides, in light of the gave scientific categorization we audit the significant interoperability taking care of procedures what's more, arrangements utilized for tending to interoperability. The review finishes by giving open research difficulties. This research work helps IoT specialists and experts distinguish the various systems for enlightening IoT interoperability to increment the number of interoperable IoT items [4].

The rest of the paper is organized as follows. Section 2, describes the problem statement. Section 3 presents the background and literature review while section 4 states the purposed model and its working. In the end, the conclusion of the study and future work is presented.

\section{Problem Statement:}

Interoperability problems for IoT rise because of the accessibility of various operating systems (OSs) with the inability to run on different devices, programming languages, data constructions, structural design, contact apparatuses for things of data.

\section{BACKGROUND AND LITERATURE REVIEW}

The issue of data framework interoperability has occurred in 1988 and conceivably much prior. There are a few descriptions for interoperability in the script. Surrounded by the differing characterizations for interoperability, we use the ones related to our specific situation. The Oxford Dictionary contributes to the ability of computer systems or programs to exchange information. This infers two interconnected frameworks communicate with each other and utilize the usefulness of one another. ISO/IEC characterizes interoperability as the capacity to impart, implement projects, or move information amid different utilitarian entities in a way that necessitates the client to have almost no learning of the interesting attributes of those entities. On a more extensive see, interoperability is characterized by the capacity of more than two or then again, more frameworks or parts to trade data and to utilize the data that has been traded. As indicated by this meaning, interoperability is acknowledged by concocting norms [5]. In IoT interoperability is characterized by the capacity of two frameworks to impart and impart administrations to each other. The capacity of two frameworks to interoperate can likewise remain displayed utilizing various kinds of layered models. For instance, a sixlevel formation including no association, specialized (essential availability and system availability), grammatical (information trade interoperability), semantic (understanding in the significance of the information), down to business, dynamic (appropriateness of the data), and calculated (shared perspective on the world) explained [6]. A comparable sixlevel model is presented having: association, correspondence, semantic, dynamic, social, and calculated. These six levels are specialized, linguistic, semantic, down to business/dynamic, reasonable, individually.

Interoperability is based on the information and technical interoperability. The part and requirement of information interoperability are absolute because there is no functional cooperation without information exchange. The protagonist of technical interoperability is very important in IoT, where technical systems and devices support almost all functional processes [7].

\section{Comparative Analysis of Existing Work}

The technical contests in communication can be defined as study creativities for discovering the opportunities of integration, interconnection, and interoperability of IoT 
technologies and structures [8]. Communication is of the highest concern as IoT allows for a heterogeneous environment in the sense of devices, services, and communication media. In the spotlight is on the issues of integration of existing protocols and technologies, scalability for new ones, keeping a good balance with the pervasiveness, context awareness, and security for IoT environments. The protocol non-interoperability can be explained through a range of real-time cases, where e.g. the diversity of the incompatible characteristics and demands for the proper functioning of the file-sharing protocols (FTP (File Transfer Protocol), NFS (Network File System), SMB (Server Message Block, Microsoft compatible), and SCP (Secure Copy Protocol) used on the application layer can disable the communication. NFS is typically used by the UNIX community and is not compatible with the MS Windows family users, while SMB stands for the MS Windows FSP and is formally no understandable for the UNIX community [9]. On the other hand, the Linux community supports both protocol types. FTP has also an open non-crypto version, and different cryptographically secured adaptations, which can additionally complicate the issue of algorithm's mutual compatibility and proper key exchange between entities. The proper IoT system functionality is potentially complicated by systems vulnerabilities to a range of cyberattacks, hence the need for security on all the layers of data/information transmission is being highly praised. It is also a foundation for the architecture and functioning of a range of security protocols related to different stack layers. Their main characteristics are the introduction of the crypto features, whereas the fundamental interoperability relies on the compatibility/understandability of the used encryption algorithms, along with the successful and secure keys exchange. The general impression is that there is a strong need for detailed and comprehensive standardization, to regulate the complex and messy results of the IoT revolution. The standardization is the main catch when considering issues with IoT like environments, and mostly targets de facto protocol layering and their interconnection in the system as a unit and on the intersystem level. When tackling the characteristics of the adopted version of the TCP/IP protocol landscape this idea is further elaborated, reaching the more fragmented structure. The aim is to provide an approach to the problem of assuring technical interoperability in the context of the needed sustainability assessment for the network/system.
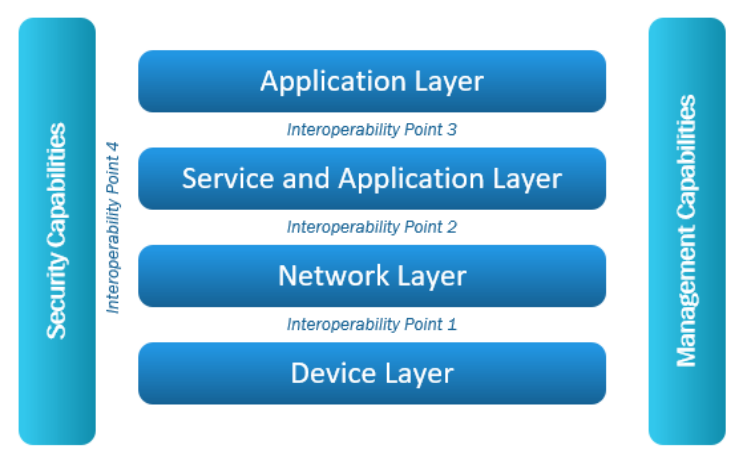

Figure1: Integration of existing protocols and interoperability points

As shown in Fig.1 IoT interoperability can be seen from alternate points of view, for example, semantic interoperability, gadget interoperability, organizing interoperability, syntactic interoperability, and stage interoperability [4].

\section{Interoperability in IoT}

To comprehend interoperability in IoT, we have to adopt a strategy for arranging it. The area of the investigation portrays an outline of IoT interoperability scientific categorization. The interoperability issues in IoT are understood from alternate points of view due to heterogeneity. Heterogeneity is 
anything but another idea nor confined to an area. Indeed, even in the corporal world, there are numerous kinds of heterogeneities, for instance, individuals talk in divergent dialects, however, they can in any case speak with one another through an interpreter (human/instruments) or by utilizing a typical language. In like manner, the various components including IoT (gadgets, correspondence, administrations, applications, and so forth.) ought to consistently coordinate and speak with one another to understand the maximum capacity of the IoT environment.

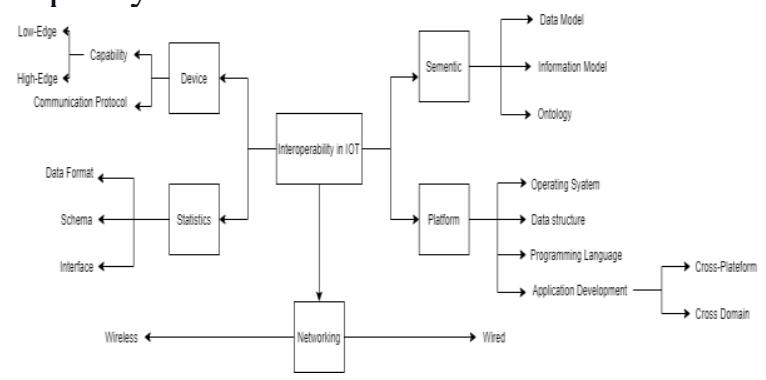

Figure 2: IoT Interoperability

\section{A. Device interoperability}

IoT is made out of an assortment of gadgets, significantly more than the customary Internet. These gadgets, which are called smart devices can comprise of top of line gadgets or low-end gadgets. The top of the line IoT gadgets have enough assets, what's more, computational abilities, for example, Raspberry $\mathrm{Pi}$ and cell phones. Then again, the low-end IoT gadgets are asset compelled as far as vitality, preparing power furthermore, correspondence capacities than normal have, for example, RFID labels, modest and minimal effort sensors, and actuators, Arduino, and Open Mote to give some examples. The microcontroller (MCU) engineering and key framework attributes of IoT gadgets, for example, processor speed, RAM, correspondence innovation, and battery limit contrast extensively between various brands and models Also, different correspondence conventions have risen because of the various prerequisites of IoT markets. For instance, IoT gadgets, for example, Smart Devices Like TV, Printers, forced air systems to bolster customary pervasive $\mathrm{Wi}-\mathrm{Fi}$ advances and 3G/4G cell correspondences. Furthermost late IoT therapeutic gadgets depend on ANT+ standard; other wearable gadgets for the most part sustenance SMART Ready also, NFC, while the natural sensors practice Zig Bee based on IEEE 802.15.4 standard. Other than these conventions, the standard correspondence conventions are used for shrewd gadgets, sensors, and actuators (i.e., Z-Wave, ZigBee, and Wireless Hart) other than the usual restrictive arrangement (i.e., LoRa, SIGFOX).

\section{B. Issues of Device Interoperability}

The compatibility of IoT devices is a crucial aspect to be considered while focusing on interoperability. Hence, standardized communication protocols need to be adapted which can be achieved through gateway-free interoperability [4]. Device security concerns are an important issue to be addressed with a new facet, to be able to distinguish various devices. The highest risk factor is that the user is unaware of the type of data being generated from their devices. The price and technology limitations restrain producers to produce interoperable devices, but if a standard is not followed, negative consequences may arise may vary according to the complexity of the systems [10]. The operating system, security, memory specifications, power specifications, size, and mobility support of IoT devices are important considerations concerning the enhancement of interoperability [11].

\section{Network interoperability}

The systems that IoT gadgets will work on will proceed to be heterogeneous, multiadministration, multi-seller and to a great extent dispersed. Not quite the same as personal computers, IoT gadgets for the most part depend on different short-run 
remote correspondence, what's more, organizing advances which is fairly more discontinuous and questionable [12]. System-level interoperability manages components to empower consistent message trade between frameworks through various (systems of systems) for start to finish correspondence. To make frameworks interoperable, every framework ought to have the option to trade messages with different frameworks through different kinds of systems. Because of the dynamic and heterogeneous system condition in IoT, the system interoperability level ought to handle challenges, for example, tending to, directing, asset enhancement, security, QoS, and versatility support [13].

\section{Syntactical interoperability}

Syntactic interoperability alludes to interoperation of the organization just as the information construction utilized in any traded data or administration among heterogeneous IoT framework substances. The interface must be characterized on behalf of every asset, uncovering some structure as indicated by some pattern. WSDL what's more, RESTful API is models. The substance of the communication should be consecutive to be sent above the network besides organization to do as such, (for example, XML or JSON). The information sender encodes information in a message utilizing syntactic guidelines, determined in syntax. The message recipient disentangles the got message utilizing syntactic standards characterized in the same or some other sentence structure. Syntactic interoperability issues emerge when the sender's encoding principles are inconsistent with the recipient's disentangling rules, which prompts crisscrossing message parse trees [14].

\section{E. Semantic interoperability}

The W3C characterizes semantic interoperability as Enabling various specialists, administrations, and applications to trading data, information, and learning in a significant manner, on and off the Web [15]. The Web OF things (WoT) addresses the present fracture by uncovering things and frameworks information and metadata through API. Yet, these endeavors have been vulnerable because the relating gatherings need to share the learning of a Programming interface and numerous gadgets don't talk a similar language, what's more, can't trade crosswise over various entryways and shrewd center points [16]. To be increasingly exact, the information produced by things about the earth may have a characterized information group (for example JSON, XML, or CSV) yet the information models and constructions utilized by various foundations are normally disparate and not continuously perfect. Plus, the information might be spoken to in different elements of estimations and comprise of additional data. This semantic contradiction between information models furthermore, data models results in IoT frameworks not ready to powerfully also consequently between work as they have various portrayals or considerations of assets furthermore, operating techniques, regardless of whether IoT frameworks uncover their information and assets to others [17].

\section{F. Existing Solutions of Semantic Interoperability}

A comprehensive and successful framework has been proposed for the creation of an ecosystem of IoT devices in a semantically interoperable manner developed upon the Thing Ecosystem Description. The accessibility of IoT devices is achieved through SPARQL which is a query-based methodology. These IoT devices then publish the diverse data which are then described through ontologies [18]. 
The use of middleware, ontologies, and semantic technologies ensure a harmonious exchange of information. Based on execution and performance assessment, tools and frameworks are suggested in This is achieved through a comparison of key semantic simulations based on the taxonomy outline [19].

Semantic interoperability enables the discovery of knowledge and grouping of different data sources. Various local structures use uniform ontologies that can be expanded to develop a meaningful approach. Hence one to one communication is not viable. Therefore, presented a mechanism for meaningful usage and interoperable system through mapping among the devices [20].

There is a need to detect a typical circumstance while pairing at the semantic interoperability. Therefore, an early warning system (ESW) needs to be devised to do the observing. The IoT-based EWS in monitors the semantic assimilation of data coming from variant devices to provide an emergency rejoinder in crucial applications respective of domain ontologies, standards, and serial arrangement. Risk assessment is done through observation of vital signs of the subject through medical devices [21].

The model proposed monitors the health status of the subjects in the healthcare environment through the incorporation of semantic Interoperability Model (IoT SIM). It relates the things with the usage of triples to form a meaningful exchange of information. Moreover, the SPARQL query is utilized for the extraction of records from the RDF graph and Tableau, Gruff -6.2.0, and MySql tools [22].

VICINITY is an H2020 design that works with the decentralization and bottom-up approach to assimilate IoT devices. It presents a transparent interoperable cloud for environments [23].

\section{G. Platform interoperability}

A cross-stage IoT application can get to diverse IoT stages and coordinate information from different stages. For instance, think about the accompanying application situation: a client who has medical issues utilizes an IoT cross-platform application consistently to assist him with his regular errands. The IoT application associates with the client's keen wellbeing stage of wearable sensors to constantly screen his wellbeing circumstances (pulse, fall circumstance, and glucose level) what's more, in a crisis, finds him and sends a rescue vehicle. The application can likewise get to a shrewd city stage to purchase a ticket to the client's wanted goal and demonstrates the quickest course to the transport/train station. The cross-stage interoperability among things and information in this situation empowers interoperability crosswise over discrete IoT stages explicit to one vertical area, for example, savvy home, brilliant medicinal services, keen greenhouse, and so forth. After cross-stage interoperability is empowered, cross-area interoperability can be accomplished in that unique stages inside heterogeneous spaces are united to manufacture flat IoT applications that demonstrate the idea behind cross-area interoperability where diverse IoT stages from various IoT areas (for example wellbeing, home, transport, and so forth.) can be incorporated to manufacture new inventive applications.

\section{H. Challenges for Platform Interoperability}

One of the key challenges related to platform interoperability of IoT is the lack of addressing all perspectives at once for the enhancement of information exchange. [24][25]. Moreover, IoT platforms should 
deem to edge computing standards for the enhancement of swiftness and competence of platforms. Correlation among IoT ecosystems in a rational and mountable manner needs to be formed to consider almost all scenarios. This should also include interoperability among the technologies irrespective of their types without having to make major alterations in their practices. Another, challenge to be highlighted is the requirement to automate interoperability testing to encourage organizations to develop interoperable solutions [27].

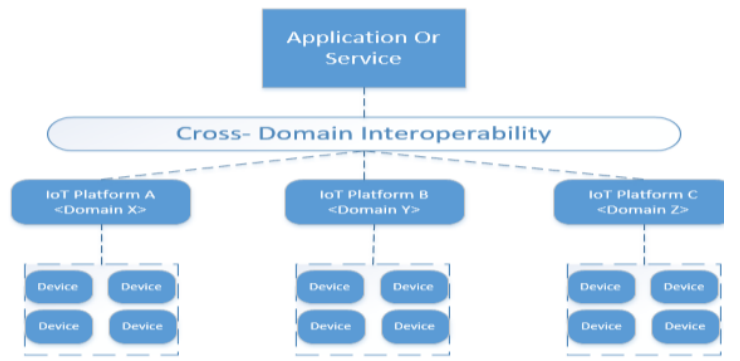

Figure 3: Cross-Domain Interoperability

To enhance the authority of IoT interoperability, analysts have utilized various methodologies, advancements which we allude to interoperability taking care of methodologies. In the accompanying, we give an outline of the distinctive interoperability taking care of approaches for tending to interoperability challenges in IoT. In specific, for every proposition, we think about the interoperability viewpoint (gadget, arrange, grammatical, semantic, cross-stage, and cross-area interoperability), interoperability methodology, receptiveness, network, application conventions, and security/ security measurements. The various recommendations are partitioned into IoT standard structures, ventures, and standards.

\section{Adapters/gateways \& Virtual networks/ overlay-based solutions}

Doors and connectors are the plans which address interoperability through the improvement of a halfway device. in some cases, called go-between to improve interoperability among IoT gadgets. The target is to connect among various details, information, measures, and middleware's and so forth. To play out a transformation between the convention of the sending gadget and the convention of the getting gadget, the passage can be extended with the utilization of modules. For instance, when IoT gadgets utilize divergent correspondence advances when they utilize divergent application layer conventions

Virtual systems or Overlay-based arrangements have been anticipated in the Bring about Ecosystems of Networked Objects^ $^{\wedge}$ (MENO), with the mean to incorporate sensors and actuators, what's more, other IP-shrewd items consistently to the Internet for start to finish correspondence [27].

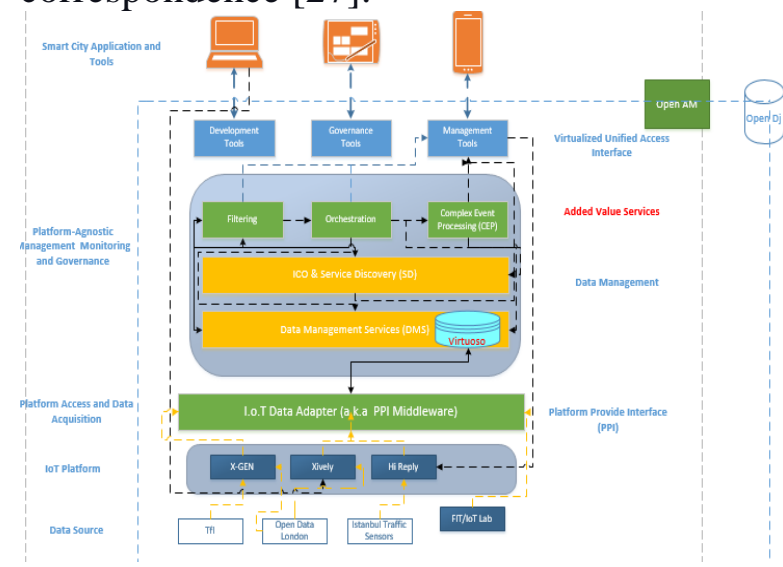

Figure 4: Adapters/gateways \& Virtual networks/ overlay-based solutions

The primary thought behind MENO is to make a virtual system over physical systems and in this manner permit correspondence with different sorts of gadgets, counting sensor hubs. Inside each virtual system, endto-end correspondence is conceivable utilizing various conventions. When start to finish correspondence is empowered, it winds up conceivable for application designers to compose new applications that 
use sensors, actuators, and different gadgets. It gives off an impression of being on track to utilize a fresh start way to deal with incorporate the physical work with the Internet in a consistent manner.

\section{Proposed Model}

\section{A. Working of Proposed Model}

1. Identification of things (Device/Service/Bio Wearable etc.)

2. Protocol Selection Based on the Structure. (Enterprise/Distributed/cloud)

a. Protocol Repository Need to be updated concerning time spam selected based on Decisions.

b. Data type identification for transmission of data between things and services with the help of relevant DATA operability APIs.

i. If data is identified requested service will be triggered.

ii. (Repository will be updated based on current successful connection.)

c. If data transmitted is identified as service Protocols will accept Virtual Machine or Sensors Linkage Based or architecture repository.

3. Services will establish requirement need

4. The service repository will be updated based on the application domain.

a. Application domain repository will be used for decision making and updating of services and architecture repository based on Things requests.

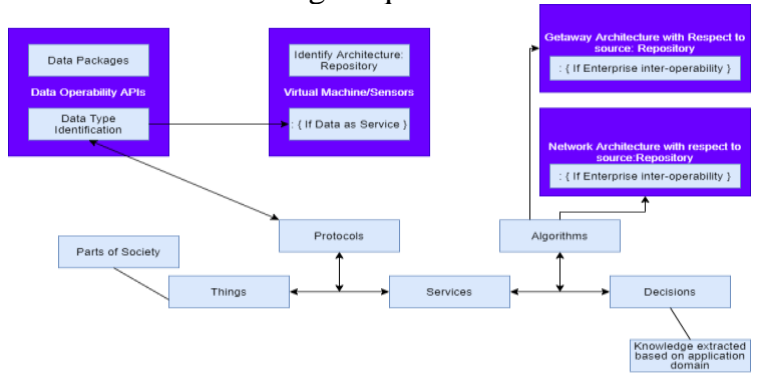

Figure 5: Decision Making based Model on Data and Services being used during Interoperability

\section{B. Keys Points of proposed Method}

a) For both Enterprise and Cloud-based Architecture

b) Required $3 \quad$ Simultaneous Repositories Relevant to Things Request.

c) Decision Making based on Data and Services being used during Inter-operability

d) Virtual Repository View for each Thing connecting to cloud-based or central data Set.

e) Reducing updating of irrelevant services.

\section{CONCLUSION}

To enhance interoperability in IoT is essential for the success of IoT. From the development of IoT, a wide range of propositions have concentrated on this urgent issue. The proposition is assorted and advances various methodologies. This paper introduces a complete outline of the subject. The scientific classification of IoT interoperability was recognized. Besides, we contemplated and arranged the associated systems for taking care of explicit kinds of interoperability. As indicated by the distinctive interoperability types furthermore, interoperability dealing with methodologies, a far-reaching study on the ongoing research has been displayed. We discussed Interoperability research issues, challenges, and prescribed conceivable upcoming research bearings. This study classified the current commendations as per their interoperability dealing with strategies: passages, virtual arrange, organizing advances, open API, System oriented architecture, semantic web innovations in addition to open guidelines. Every classification with numerous interoperability proposition, the major is discussed in the paper. Simply it would be difficult to separate all linked IoT propositions and stages. 


\section{Future Work:}

The absence of benchmarks and nonappearance of front-line innovations moderates the advancement of IoT. Giving semantically interoperable stages over the distinctive IoT spaces has a reasonable prerequisite for research upgrades.

\section{References}

[1] Vazirani, S. (2018). A Review Paper On Iot \& Its Smart Applications.

[2] Sarker, S., Roy, K., Afroz, F., \& Pathan, A. S. K. (2020). On the Opportunities, Applications, and Challenges of Internet of Things. In Decentralised Internet of Things (pp. 231-254). Springer, Cham

[3] S. Ben Mokhtar, N. Georgantas, and V. Issarny, 'COCOA: Conversationbased service composition in pervasive computing environments with QoS support," J. Syst. Softw., vol. 80, no. 12, pp. 1941_1955, 2007]

[4] Noura, M., Atiquzzaman, M. \& Gaedke, M. Interoperability in Internet of Things: Taxonomies and Open Challenges. Mobile Netw Appl 24, 796809 (2019).]

[5] Kubicek, H., Cimander, R., \& Scholl, H. J. (2011). Organizational interoperability in e-government: lessons from 77 European good-practice cases. Springer Science \& Business Media.]

[6] Tolk, A., \& Muguira, J. A. (2003, September). The levels of conceptual interoperability model. In Proceedings of the 2003 fall simulation interoperability workshop (Vol. 7, pp. 1-11). Citeseer.]

[7] Sándor, M. U. N. K. (2002). An analysis of basic interoperability related terms, system of interoperability types. ACADEMIC AND APPLIED RESEARCH IN MILITARY SCIENCE I.:(1) pp. 117-132.(2002)

[8] Nicolescu, R., Huth, M., Radanliev, P. et al. Mapping the values of IoT. J Inf Technol 33, 345-360 (2018). https://doi.org/10.1057/s41265-0180054-1

[9] Lee, S. K., Bae, M., \& Kim, H. (2017). Future of IoT networks: A survey. Applied Sciences, 7(10), 1072

[10] Mridha, M. F., Hamid, M. A., \& Asaduzzaman, M. (2020). Issues of Internet of Things (IoT) and an intrusion detection system for IoT using machine learning paradigm. In Proceedings of International Joint Conference on Computational Intelligence (pp. 395406). Springer, Singapore

[11] .Ojo, M.O., Giordano, S., Procissi, G., \& Seitanidis, I.N. (2018). A Review of Low-End, Middle-End, and High-End Iot Devices. IEEE Access, 6, 7052870554.

[12] Sethi, P., \& Sarangi, S. R. (2017). Internet of things: architectures, protocols, and applications. Journal of Electrical and Computer Engineering, 2017

[13] López, Diego \& Blobel, Bernd. (2008). A development framework for semantically interoperable health information systems. International journal of medical informatics. 78. 83103. 10.1016/j.ijmedinf.2008.05.009

[14] Sudmanns, M., Tiede, D., Lang, S., \& Baraldi, A. (2018). Semantic and syntactic interoperability in online 
processing of big Earth observation data. International journal of digital earth, 11(1), 95-112.

[15] Iftikhar, Saman \& Khan, Wajahat \& Ahmad, Hafiz \& Fatima, Kiran. (2012). Semantic Interoperability in E-Health for Improved Healthcare. 10.5772/36469

[16] Thakuriah, P., \& Geers, D. G. (2013). Transportation and information: trends in technology and policy. New York: Springer.

[17] Amoaha, E. K., \& Nguyenb, T. V. (2019). Optimizing the Usage of Building Information Model (BIM) Interoperability Focusing on Data Not Tools. In ISARC. Proceedings of the International Symposium on Automation and Robotics in Construction (Vol. 36, pp. 1081-1090). IAARC Publications

[18] Cimmino, A., Poveda-Villalón, M., \& García-Castro, R. (2020). eWoT: A Semantic Interoperability Approach for Heterogeneous IoT Ecosystems Based on the Web of Things. Sensors, 20(3), 822.

[19] Rahman, H., \& Hussain, M. I. (2019). A comprehensive survey on semantic interoperability for Internet of Things: State-of-the-art and research challenges. Transactions on Emerging Telecommunications Technologies.

[20] Lakka, E., Petroulakis, N. E., Hatzivasilis, G., Soultatos, O., Michalodimitrakis, M., Rak, U., ... \& Kulkarni, V. (2019, September). End-toEnd Semantic Interoperability Mechanisms for IoT. In 2019 IEEE 24th International Workshop on Computer Aided Modeling and Design of
Communication Links and Networks (CAMAD) (pp. 1-6). IEEE

[21] Moreira, J., Pires, L. F., van Sinderen, M., Wieringa, R., Singh, P., Costa, P. D., \& Llop, M. (2019). Improving the semantic interoperability of IoT Early Warning Systems: the Port of Valencia use case. In Enterprise Interoperability VIII (pp. 17-29). Springer, Cham.

[22] Jabbar, S., Ullah, F., Khalid, S., Khan, M., \& Han, K. (2017). Semantic interoperability in heterogeneous IoT infrastructure for healthcare. Wireless Communications and Mobile Computing, 2017

[23] Cimmino, A., Oravec, V., Serena, F., Kostelnik, P., Poveda-Villalón, M., Tryferidis, A., ... \& Grimm, C. (2019, May). VICINITY: IoT Semantic Interoperability Based on the Web of Things. In 2019 15th International Conference on Distributed Computing in Sensor Systems (DCOSS) (pp. 241-247). IEEE

[24] Medagliani, Paolo \& Leguay, Jeremie \& Duda, A. \& Rousseau, Franck \& Duquennoy, Simon \& Raza, Shahid \& Ferrari, Gianluigi \& Gonizzi, Pietro \& Cirani, Simone \& Veltri, L. \& Montón, Màrius \& Domingo Prieto, Marc \& Dohler, M. \& Villajosana, I. \& Dupont, O.. (2014). Internet of Things Applications - From Research and Innovation to Market Deployment.

[25] M. R. Naqvi, M. Aslam, M. W. Iqbal, S. Khuram Shahzad, M. Malik and M. U. Tahir, "Study of Block Chain and its Impact on Internet of Health Things (IoHT):Challenges and Opportunities," 
2020 International Congress on HumanComputer Interaction, Optimization and Robotic Applications (HORA), Ankara, Turkey, 2020, pp. 1-6, doi: 10.1109/HORA49412.2020.9152846.

[26] M. R. Naqvi, M. Arfan Jaffar, M. Aslam, S. K. Shahzad, M. Waseem Iqbal and A. Farooq, "Importance of Big Data in Precision and Personalized Medicine," 2020 International Congress on HumanComputer Interaction, Optimization and Robotic Applications (HORA), Ankara, Turkey, 2020, pp. 1-6, doi: 10.1109/HORA49412.2020.9152842.

[27] Ullah, Israr, Shakeel Ahmad, Faisal Mehmood, and DoHyeun Kim. "Cloud Based IoT Network Virtualization for Supporting Dynamic Connectivity among Connected Devices." Electronics 8, no. 7 (2019): 742. 